\title{
Greater than 3-Log Reduction in Viable Coronavirus Aerosol Concentration in Ducted Ultraviolet-C (UV-C) Systems
}

\author{
Yuechen Qiao ${ }^{* * *}$, My Yang ${ }^{2 * *}$, Ian A. Marabella ${ }^{1}$, Devin A. J. McGee ${ }^{1}$, Hamada Aboubakr², \\ Sagar Goyal ${ }^{2}$, Christopher J. Hogan Jr. ${ }^{1},$, Bernard A. Olson ${ }^{1 * *},{ }^{1 *}$ Montserrat Torremorell ${ }^{2 * *}$ \\ ${ }^{1}$ Department of Mechanical Engineering, University of Minnesota, Minneapolis, MN 55455 \\ ${ }^{2}$ Department of Veterinary Population Medicine, College of Veterinary Medicine, \\ University of Minnesota, Saint Paul, MN 55108, USA
}

Summary of contents ( 3 pages total):

- Table S1. Oligonucleotides for PRCV RT-qPCR used in this study.

- Figure S1. The size distribution functions for the DMEM solution nebulized by number (a) and by volume (b). Results are based on combined measurements from an SMPS and APS. Particles are assumed spherical with unit density in APS data interpretation.

- Standard Deviation of the Log Reduction 
$R T$-qPCR primers

Table S1. Oligonucleotides for PRCV RT-qPCR used in this study.

\begin{tabular}{|c|c|c|c|c|c|}
\hline $\begin{array}{c}\text { Name of Primer } \\
\text { or Probe }\end{array}$ & $\begin{array}{c}\text { Oligonucleotide } \\
\text { name }\end{array}$ & Sequence $\left(5\right.$, à3') ${ }^{a}$ & Polarity $^{b}$ & Position & $\begin{array}{l}\text { Product } \\
\text { length } \\
\text { (bp) }\end{array}$ \\
\hline \multirow{3}{*}{ PRCV RT-qPCR } & PRCV-20-F & TGCTAGTTATGTRGCTAATGTY & + & $20436-20457^{\mathbf{c}}$ & \multirow{3}{*}{112} \\
\hline & PRCV- 21-R & ACCACTAACCAACGTGGARC & - & $20528-20547 \mathbf{c}$ & \\
\hline & PRCV-22-P & 56-JOEN/ACVMARMCA/ZEN/GGAGGYTTTATACCA/ 3IABkFQ & + & $20464-20487^{\mathbf{c}}$ & \\
\hline \multirow{2}{*}{$\begin{array}{l}\text { PRCV RT-PCR } \\
\text { (For standard curve } \\
\text { construction) }\end{array}$} & PRCV-23-F & TTGTGGTTTTKGKYRTAATGCCH & + & $20366-20388^{\mathbf{c}}$ & \multirow{2}{*}{509} \\
\hline & PRCV-24-R & CGWAACAGTACCGTGGTCCA & - & $20856-20875^{\mathbf{c}}$ & \\
\hline
\end{tabular}

a Mixed bases in degenerate primers and probes are as follows: $\mathrm{H} \approx \mathrm{A}$ or $\mathrm{C}$ or $\mathrm{T} ; \mathrm{K} \approx \mathrm{G}$ or $\mathrm{T} ; \mathrm{M} \approx \mathrm{A}$ or $\mathrm{C} ; \mathrm{N} \approx$ any; $\mathrm{R} \approx \mathrm{A}$ or $\mathrm{G} ; \mathrm{V} \approx \mathrm{A}$ or $\mathrm{C}$ or $\mathrm{G} ; \mathrm{W} \approx \mathrm{A}$ or $\mathrm{T} ; \mathrm{Y} \approx \mathrm{C}$ or $\mathrm{T}$.

$\mathrm{b}+$, virus sense; - , anti-virus sense.

c Corresponding nucleotide position of PRCV (GenBank accession no.: KY406735.1) as reference.

\section{Particle Size Distribution Functions}
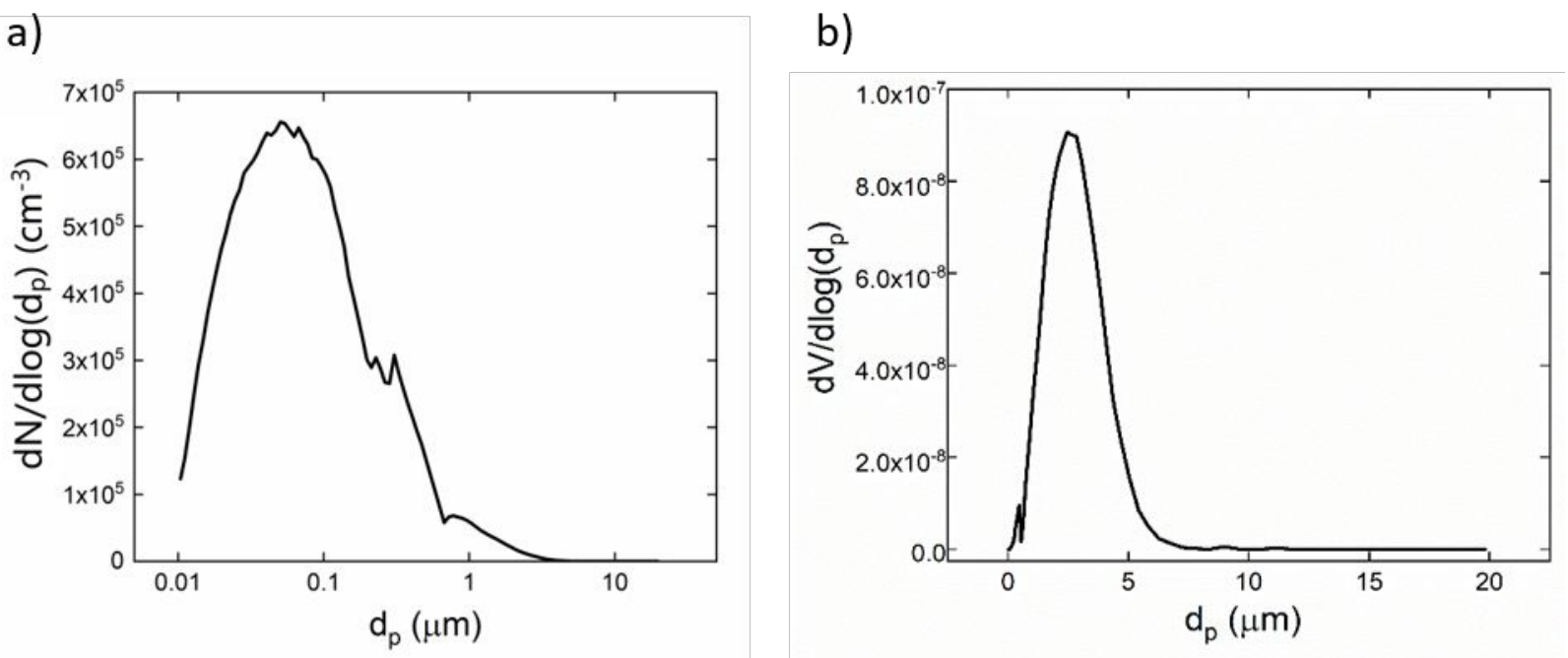

Figure S1. The size distribution functions for the DMEM solution nebulized by number (a) and by volume (b). Results are based on combined measurements from an SMPS and APS. Particles are assumed spherical with unit density in APS data interpretation.

Standard Deviation of the Log Reduction 
As noted in the main text, log reduction is computed via the equation:

$$
L R=\log _{10}\left(\frac{C_{u p}}{C_{\text {down }}}\right)
$$

The standard deviation on the log reduction, $\sigma_{L R}$, is estimated via the root-sum-square (RSS) method using the equation:

$$
\begin{aligned}
\sigma_{L R} & =\sqrt{\left(\frac{\partial L R}{\partial C_{u p}}\right)^{2} \sigma_{u p}^{2}+\left(\frac{\partial L R}{\partial C_{\text {down }}}\right)^{2} \sigma_{d o w n}^{2}} \\
\sigma_{L R} & =\frac{1}{2.30} \sqrt{\left(\frac{\sigma_{u p}}{C_{u p}}\right)^{2}+\left(\frac{\sigma_{\text {down }}}{C_{\text {down }}}\right)^{2}}
\end{aligned}
$$

where $\sigma_{u p}$ and $\sigma_{\text {down }}$ are the standard deviations of the upstream and downstream triplicate measurements. 